\title{
ON SYSTEM OF PARAMETERS, LOCAL INTERSECTION MULTIPLICITY AND BEZOUT'S THEOREM
}

\author{
EDUARD BODA AND WOLFGANG VOGEL ${ }^{1}$
}

\begin{abstract}
This paper provides effective methods for computing the local intersection multiplicity as the length of a well-defined ideal (see Theorem and Proposition 1). There are other ways of obtaining such an ideal (see [2], [9], [12], [18]) but ours is simpler because of our use of reducing systems of parameters. Applying these ideal theoretic methods we will give a new and simple proof of Bezout's Theorem (see \$4). Hence this proof again provides the connection between the different viewpoints which are treated in the work of Lasker-Macaulay-Gröbner and Severi-van der Waerden-Weil concerning the multiplicity theory.
\end{abstract}

1. Introduction. Let $X, Y$ be subvarieties of projective space $\mathbf{P}_{k}^{n}$ over a fixed algebraically closed field $K$. If $X$ and $Y$ intersect properly, and $C$ is an irreducible component of $X \cap Y$, we denote by $i(X, Y ; C)$ the local intersection multiplicity of $X$ and $Y$ along $C$ defined by A. Weil [17]. Let $A$ be the local ring of the generic point of $C$ on $\mathbf{P}_{K}^{n}$, and $\mathfrak{a}$ and $\mathfrak{b}$ the ideals of $X$ and $Y$ in $A$. Applying the reduction to the diagonal there is no loss of generality in assuming that one variety, say $Y$, is a complete intersection. Then we know that the multiplicity $i(X, Y ; C)$ is given by the multiplicity of $\mathfrak{a}+\mathfrak{b} / \mathfrak{a}$ with respect to $A / \mathfrak{a}$ (notation: $e_{0}(\mathfrak{b}, A / \mathfrak{a})$ ) where $e_{0}(\mathfrak{b}, A / \mathfrak{a})$ is the coefficient of the $\left(\begin{array}{c}n+\operatorname{dim} A / \mathfrak{a} \\ \operatorname{dim} A / \mathfrak{a}\end{array}\right)$ term in the Hilbert-Samuel polynomial $P(n ; A / \mathfrak{a}, \mathfrak{b})$, see [10, Chapter II, \$7.b]. The first example which showed that in general the length of $A / \mathfrak{a}+\mathfrak{b}$ is not $e_{0}(\mathfrak{b}, A / \mathfrak{a})$ is given by B. L. van der Waerden [16] by using Macaulay's famous space curve, given parametrically by

$$
\left\{u^{4}, u^{3} v, u v^{3}, v^{4}\right\} \quad(\text { see }[5, \text { p. 98]). }
$$

We know that $e_{0}(\mathfrak{b}, A / \mathfrak{a})$ is given by the naive definition, taking the length of $A / \mathfrak{a}+\mathfrak{b}$, if and only if $A / \mathfrak{a}$ is a Cohen-Macaulay ring, see e.g. [19]. It is precisely this phenomenon which makes this naive definition, modeled after the case of curves on a surface, uninteresting.

However, the aim of this note is to give effective methods for computing the local intersection multiplicity as the length of $A / \mathrm{c}$ where $\mathrm{c}$ is a well-defined ideal of $A /$ a (see Theorem and Proposition 1). There are other ways of obtaining such an ideal (see [2], [9], [12], [18]) but ours is simpler because of

Received by the editors April 11, 1978 and, in revised form, June 20, 1978.

AMS (MOS) subject classifications (1970). Primary 13H15; Secondary 14B99.

Key words and phrases. Local intersection multiplicity, system of parameters, reducing system of parameters, Cohen-Macaulay rings, Buchsbaum rings and Bezout's Theorem.

${ }^{1}$ The second author was supported by the National Science Foundation at Brandeis University. 
our use of reducing systems of parameters. Using the above notations we note that $\mathfrak{c}=\mathfrak{a}+\mathfrak{b}$ if and only if $A / \mathfrak{a}$ is a Cohen-Macaulay ring. Applying these methods we will give a new and simple proof of Bezout's Theorem (see §4). In $\$ 5$ we conclude by studying some examples and add some remarks.

The authors are indebted to D. Eisenbud for helpful discussions and the referee for pertinent remarks concerning this manuscript.

2. Results. We fix the following notations. Let $A$ be a commutative Noetherian local ring of dimension $d \geqslant 1$. The maximal ideal of $A$ will be denoted throughout by $m$. The dimension of an ideal a of $A$, written $\operatorname{dim}(\mathfrak{a})$, is the (Krull) dimension of $A / \mathfrak{a}$. The multiplicity of an m-primary ideal $q$ is denoted $e_{0}(\mathfrak{q}, A)$; the length of the $A$-module $A / \mathfrak{q}$ is denoted $l(A / q)$ (see e.g. [19]). For an ideal $a$ of $A$ we set $U(\mathfrak{a})=\cap q$ where $q$ runs through the primary ideals belonging to a such that $\operatorname{dim}(q)=\operatorname{dim}(a)$.

Let $q$ be generated by the system of parameters $a_{1}, \ldots, a_{d}$. Then we put $a_{0}=(0)$ and $a_{k}=\left(a_{k}\right)+U\left(a_{k-1}\right)$ for any $0<k<d$. Using this algorithm we get

Proposition 1. $e_{0}(\mathfrak{q}, A)=l\left(A / \mathfrak{a}_{d}\right)$

Our main result is the following theorem.

THEOREM. Let a be generated by the system of parameters $a_{1}, \ldots, a_{d}$ in $A$. Then the following statements are equivalent:

(i) $e_{0}(\mathfrak{q}, A)=l\left(A /\left(a_{d}\right)+U\left(\left(a_{1}, \ldots, a_{d-1}\right)\right)\right)$.

(ii) $a_{j}$ is not in any prime $\mathfrak{p}$ belonging to $\left(a_{1}, \ldots, a_{j-1}\right)$, such that $\operatorname{dim}(\mathfrak{p})=d$ - j for any $j=1, \ldots, d-1$.

(iii) $U\left(a_{k}\right)=U\left(\left(a_{1}, \ldots, a_{k}\right)\right)$ for every $k=0,1, \ldots, d-1$.

(iv) $U\left(a_{d-1}\right)=U\left(\left(a_{1}, \ldots, a_{d-1}\right)\right)$.

In the language of $\left[1\right.$, p. 643], (ii) above asserts that $a_{1}, \ldots, a_{d}$ is a reducing system of parameters. The following proposition makes this theorem useful and strengthens Proposition 4.9 of [1].

Proposition 2. Let $\mathfrak{q} \subset A$ be an ideal generated by a system of parameters. Then $q$ can be generated by the elements $b_{1}, \ldots, b_{d}$ such that $b_{k}$ is not in any prime $\mathfrak{p} \neq \mathrm{m}$ belonging to $\left(b_{1}, \ldots, b_{k-1}\right)$ for any $k=1, \ldots, d$.

\section{Proofs.}

Proof of Proposition 1. It is known that (see for example Theorem 6, Chapter 7 in [7])

$$
e_{0}(q, A)=e_{0}\left(\left(a_{2}, \ldots, a_{d}\right), A /\left(\left(a_{1}\right)+0: a_{1}^{n}\right)\right)
$$

for large $n$. In order to prove the proposition we will use induction on $d$. Let $d=1$. We obtain

$$
\begin{aligned}
e_{0}\left(\left(a_{1}\right), A\right) & =l\left(A /\left(a_{1}\right)+0: a_{1}^{n}\right)=l\left(A /\left(a_{1}\right)+U(0)\right) \text { since } n \gg 0 \\
& =l\left(A / a_{1}\right) .
\end{aligned}
$$


Let $d>1$. We get

$$
e_{0}(q, A)=e_{0}\left(\left(a_{2}, \ldots, a_{d}\right), A /\left(a_{1}\right)+0: a_{1}^{n}\right) .
$$

We set $A^{\prime}=A /\left(a_{1}\right)+0: a_{1}^{n}$. We can apply the induction to $\left(a_{2}, \ldots, a_{d}\right)$ in $A^{\prime}$. We put $\mathfrak{a}_{1}^{\prime}=(0)$ in $A^{\prime}$ and $\mathfrak{a}_{k}^{\prime}=\left(a_{k}\right)+U\left(\mathfrak{a}_{k-1}^{\prime}\right)$ for any $1<k \leqslant d$. Therefore we obtain that

$$
e_{0}(\mathfrak{q}, A)=l\left(A^{\prime} / \mathfrak{a}_{d}^{\prime}\right)
$$

We still have to show that

$$
U\left(\left(a_{1}\right)+0: a_{1}^{n}\right)=U\left(\left(a_{1}\right)+U(0)\right) \text { for large } n .
$$

From this follows the proposition since $a_{1}=\left(a_{1}\right)+U(0)$. First we will show that $\left(a_{1}\right)+0: a_{1}^{n}$ and $\left(a_{1}\right)+U(0)$ have the same associated prime ideals of dimension $d-1$. It suffices to check this after localizing at such a prime ideal of dimension $d-1$ that contains $a_{1}$. But then $\left(a_{1}\right)$ contains a power of the maximal ideal, so 0: $a_{1}^{n}=U(0)$ for large $n$ (see also [2, Theorem 1]).

Considering the localizations of the ideals $\left(a_{1}\right)+U(0)$ and $\left(a_{1}\right)+0: a_{1}^{n}$ at the prime ideals of dimension $d-1$ which belong to $\left(a_{1}\right)+U(0)$ we get our statement (*). Q.E.D.

In order to prove the theorem we give the following lemma.

LEMMA. Let $q$ be generated by the system of parameters $a_{1}, \ldots, a_{d}$ in $A$. If an integer $n$ is sufficiently large we have

$$
U\left(\left(a_{1}, \ldots, a_{d-1}\right)\right) \cap\left(a_{1}, \ldots, a_{d-1}, a_{d}^{n}\right)=\left(a_{1}, \ldots, a_{d-1}\right) .
$$

Proof. We will show that

$$
U(0) \cap\left(a_{d}^{n}\right)=(0) \text { in } A /\left(a_{1}, \ldots, a_{d-1}\right) .
$$

We will work in the ring $A /\left(a_{1}, \ldots, a_{d-1}\right)$. Suppose now that $n$ is large enough to ensure that $U(0)=(0): a_{d}^{n}$. Hence we get

$$
\begin{aligned}
U(0) \cap\left(a_{d}^{n}\right) & =\left((0):\left(a_{d}^{n}\right)\right) \cap\left(a_{d}^{n}\right) \\
& =\left((0):\left(a_{d}^{n}\right)\right) \cdot\left(a_{d}^{n}\right) \text { because } a_{d} \text { is a non-zero-divisor on } A / U(0) \\
& =(0) \text { Q.E.D. }
\end{aligned}
$$

Proof of The Theorem. (i) $\Rightarrow$ (ii): We put $q^{\prime}=\left(a_{1}, \ldots, a_{d-1}, a_{d}^{n}\right)$ for $n \gg 0$ and $a_{d-1}=\left(a_{1}, \ldots, a_{d-1}\right)$. Since $a_{d}$ is a non-zero-divisor on $A / U\left(q_{d-1}\right)$ we obtain

$$
\begin{aligned}
n \cdot e_{0}(\mathfrak{q}, A) & =e_{0}\left(\mathfrak{q}^{\prime}, A\right)=l\left(A /\left(a_{d}^{n}\right)+U\left(\mathfrak{q}_{d-1}\right)\right) \\
& =l\left(A / \mathfrak{q}^{\prime}\right)-l\left(\left(a_{d}^{n}\right)+U\left(\mathfrak{q}_{d-1}\right) / \mathfrak{q}^{\prime}\right) \\
& =l\left(A / \mathfrak{q}^{\prime}\right)-l\left(U\left(\mathfrak{q}_{d-1}\right) / \mathfrak{q}^{\prime} \cap U\left(\mathfrak{q}_{d-1}\right)\right) \\
& =l\left(A / \mathfrak{q}^{\prime}\right)-l\left(U\left(\mathfrak{q}_{d-1}\right) / \mathfrak{q}_{d-1}\right), \quad \text { by the lemma, } \\
& =l\left(A / \mathfrak{q}^{\prime}\right)-l\left(\mathfrak{q}_{d-1}: a_{d}^{n} / \mathfrak{q}_{d-1}\right), \quad \text { since } n \gg 0 .
\end{aligned}
$$

Hence Corollary 4.8 of [1] yields the statement (ii).

(ii) $\Rightarrow$ (iii): We will use induction on $k$. If $k=0$ then the assertion is trivial. Suppose $U\left(a_{k}\right)=U\left(\left(a_{1}, \ldots, a_{k}\right)\right)$ for any $0<k<d-1$. Since 
$\left(a_{1}, \ldots, a_{k+1}\right) \subseteq a_{k+1}$ and $\operatorname{dim}\left(\left(a_{1}, \ldots, a_{k+1}\right)\right)=\operatorname{dim}\left(a_{k+1}\right)$ we get

$$
U\left(\left(a_{1}, \ldots, a_{k+1}\right)\right) \subseteq U\left(a_{k+1}\right) .
$$

We now show that $U\left(\left(a_{1}, \ldots, a_{k}\right)\right) \subseteq U\left(\left(a_{1}, \ldots, a_{k}, a_{k+1}\right)\right)$. Take an element $x \notin U\left(\left(a_{1}, \ldots, a_{k+1}\right)\right)$. Then there is a prime ideal $\mathfrak{p}_{0}$ belonging to $\left(a_{1}, \ldots, a_{k+1}\right)$ with $\operatorname{dim}\left(\mathfrak{p}_{0}\right)=d-k-1$ such that we have

$$
\left(a_{1}, \ldots, a_{k}\right): x \subseteq\left(a_{1}, \ldots, a_{k+1}\right): x \subseteq \mathfrak{p}_{0} .
$$

By assumption $\mathfrak{p}_{0}$ cannot belong to $\left(a_{1}, \ldots, a_{k}\right)$ since $a_{k+1} \in \mathfrak{p}_{0}$. There is a prime ideal $\mathfrak{p}_{1}$ belonging to $\left(a_{1}, \ldots, a_{k}\right)$ such that

$$
\left(a_{1}, \ldots, a_{k}\right): x \subseteq \mathfrak{p}_{1} \subset \mathfrak{p}_{0} .
$$

Hence we get $\operatorname{dim}\left(\mathfrak{p}_{1}\right)=d-k$; that is $x \notin U\left(\left(a_{1}, \ldots, a_{k}\right)\right)$.

Using the induction hypothesis we therefore obtain

$$
\begin{aligned}
a_{k+1} & =\left(a_{k+1}\right)+U\left(a_{k}\right)=\left(a_{k+1}\right)+U\left(\left(a_{1}, \ldots, a_{k}\right)\right) \\
& \subseteq\left(a_{k+1}\right)+U\left(\left(a_{1}, \ldots, a_{k+1}\right)\right)=U\left(\left(a_{1}, \ldots, a_{k+1}\right)\right) .
\end{aligned}
$$

Thus it follows $U\left(a_{k+1}\right) \subseteq U\left(\left(a_{1}, \ldots, a_{k+1}\right)\right)$ and the statement (iii) is proved.

(iii) $\Rightarrow$ (iv) is trivial.

(iv) $\Rightarrow$ (i): This follows from Proposition 1. Q.E.D.

Proof of Proposition 2. We set $q=\left(a_{1}, \ldots, a_{d}\right)$. Let $i$ be an integer with $1 \leqslant i \leqslant d$. Suppose there exist elements $b_{1}, \ldots, b_{i-1}$ such that $q=$ $\left(b_{1}, \ldots, b_{i-1}, a_{i}, \ldots, a_{d}\right)$ and $b_{j}$ is not in any prime $\mathfrak{p} \neq \mathfrak{m}$ belonging to $\left(b_{1}, \ldots, b_{j-1}\right)$ for any $j=1, \ldots, i-1$. We set $a_{i}=\left(b_{1}, \ldots, b_{i-1}\right.$, $\left.a_{i+1}, \ldots, a_{d}\right)$. We note that $\mathfrak{q} \underline{\mathrm{Z}} \mathrm{m} \cdot \mathfrak{q}+\mathfrak{q}_{i}$. Hence there is an element $b_{i} \in \mathfrak{q}$ such that $b_{i} \notin \mathfrak{m} \cdot \mathfrak{q}+\mathfrak{q}_{i}$ and $b_{i} \notin \mathfrak{p}$ for any $\mathfrak{p} \neq \mathfrak{m}$ belonging to $\left(b_{1}, \ldots, b_{i-1}\right)$. Since $b_{1}, \ldots, b_{i}, a_{i+1}, \ldots, a_{d}$ are linear independent mod $\mathrm{m}$. q, Nakayama's lemma implies $q=\left(b_{1}, \ldots, b_{i-1}, b_{i}, a_{i+1}, \ldots, a_{d}\right)$. Q.E.D.

4. Bezout's Theorem. Applying the above methods we will giv $:$ a new proof of the following theorem.

Bezout's THEOREM. Let $K$ be an arbitrary algebraically closed field. Let $X$, $Y$ be two subvarieties of $\mathbf{P}_{K}^{n}$ of pure dimension such that $\operatorname{dim}(X \cap Y)=$ $\operatorname{dim}(X)+\operatorname{dim}(Y)-n$. Then

$$
\operatorname{deg}(X) \cdot \operatorname{deg}(Y)=\sum_{C} i(X, Y ; C) \cdot \operatorname{deg}(C)
$$

where $C$ runs through the irreducible components of $X \cap Y$ with $\operatorname{dim}(C)=$ $\operatorname{dim}(X \cap Y)$.

Proof. In order to prove Bezout's Theorem there is no loss of generality in assuming that one variety, say $Y$, is a complete intersection. One does this by replacing $X$ by the join, $X^{\prime}$, in $P^{2 n+1}$, of $X$ and $Y$ regarded as embedded in disjoint linear subspaces of dimension $n$, and $Y$ by a linear subspace $Y^{\prime}$ of dimension $n$ which is disjoint from each. One checks algebraically, by considering homogeneous ideals, that the local intersection multiplicities of $X^{\prime}$ and $Y^{\prime}$ are the same as those of $X$ and $Y$, see [17]. We fix the following 
notations: Let $\mathfrak{a}, \mathfrak{b}=\left(F_{1}, \ldots, F_{t}\right)$ and $\mathfrak{p}_{C}$ be the defining ideals of $X, Y$ and $C$ in $K\left[x_{0}, x_{1}, \ldots, x_{n}\right]=: R$. Then $\operatorname{dim}(X \cap Y)=\operatorname{dim}(X)-t$, and the degree of $X$ is given by $h_{0}(a)$ where $h_{0}$ is the first coefficient of the Hilbert polynomial of $a$. We put in $R: a_{0}=a$ and $a_{k}=\left(F_{k}\right)+U\left(a_{k-1}\right)$ for any $0<k<t$. Then we prove our first claim.

Claim 1. $h_{0}\left(a_{t}\right)=h_{0}(\mathfrak{a}) \cdot h_{0}(\mathfrak{b})$.

Proof. $h_{0}\left(a_{t}\right)=h_{0}\left(\left(F_{t}\right)+U\left(a_{t-1}\right)\right)=h_{0}\left(U\left(a_{t-1}\right)\right) \cdot h_{0}\left(\left(F_{t}\right)\right)$ since $U\left(a_{t-1}\right)$ : $F_{t}=U\left(a_{t-1}\right)$. This follows, for example from [4, 143.7].

Now, it is $h_{0}\left(U\left(a_{t-1}\right)\right)=h_{0}\left(a_{t-1}\right)$ (see e.g. $\left.[4,143.1]\right)$. Therefore we get that $h_{0}\left(a_{t}\right)=h_{0}\left(\left(F_{1}\right)\right) \cdots \cdot h_{0}\left(\left(F_{t}\right)\right) \cdot h_{0}(\mathfrak{a})=h_{0} \cdot(\mathfrak{a}) h_{0}(\mathfrak{b})$ since $Y$ is a complete intersection (see e.g. $[4,142.4])$.

Claim 2. $h_{0}\left(a_{t}\right)=\Sigma_{C} i(X, Y ; C) \cdot \operatorname{deg}(C)$.

Proof. First we note that $a_{t}$ and $a+b$ have the same associated prime ideals of $\operatorname{dim}(X \cap Y)$. A simple induction on $t$ will give this result by applying Krull's principal ideal theorem. Let $q_{C}$ be the associated primary ideal of $a_{t}$ which belongs to $\mathfrak{p}_{C}$. Then it follows (see e.g. [4, 143.1 and 143.5]) that

$$
h_{0}\left(\mathfrak{a}_{t}\right)=\sum_{C} l\left(R_{\mathfrak{p}_{C}} / \mathfrak{q}_{C} R_{\mathfrak{p}_{C}}\right) \cdot h_{0}\left(\mathfrak{p}_{C}\right)
$$

Now, $l\left(R_{\mathfrak{p}_{c}} / \mathfrak{a}_{C} R_{\mathfrak{p}_{c}}\right)=l\left((R / \mathfrak{a})_{\mathfrak{p}_{c}} / \mathfrak{a}_{t}(R / \mathfrak{a})_{\mathfrak{p}_{c}}\right)$. We consider an ideal generated by the system of parameters $F_{1}, \ldots, F_{t}$ in $(R / a)_{\mathfrak{p}_{c}}=: R^{\prime}$. We set $\mathfrak{b}_{0}=(0)$ in $R^{\prime}$ and $\mathfrak{b}_{k}=\left(F_{k}\right)+U\left(\mathfrak{b}_{k-1}\right)$ for any $0<k<t$ in $R^{\prime}$. Then we get that $\mathfrak{b}_{t}=\mathfrak{a}_{t} \cdot R^{\prime}$. Hence Proposition 1 and the Theorem of reduction of $P$. Samuel [10, Chapter II, §7] yield that $l\left(R_{\mathfrak{p}_{C}} / \mathfrak{q}_{C} R_{\mathfrak{p}_{c}}\right)=i(X, Y ; C)$. Q.E.D.

5. Remarks and examples. (1) Let $A$ be a local ring such that each system of parameters is a reducing system of parameters. Such local rings yield a generalization of the (local) Buchsbaum rings and were studied in [3]. For example, let $X$ be a locally Cohen-Macaulay projective variety, and let $A$ be the local ring of the vertex of the affine cone over $X$. Then we know that each system of parameters of $A$ is a reducing system of parameters. Hence we can apply our Theorem to $A$ for each system of parameters. We have this situation in the following example.

(2) ExAmple. Take the classical example from [16, §11] (see also [4, p. 180] and [6, p. 126]). We use the notation from the introduction. Let $X, Y$ and $C$ be the subvarieties of $\mathbf{P}_{K}^{4}$ with defining prime ideals

$$
\begin{aligned}
& \mathfrak{p}_{X}=\left(x_{1} x_{4}-x_{2} x_{3}, x_{1}^{2} x_{3}-x_{2}^{3}, x_{1} x_{3}^{2}-x_{2}^{2} x_{4}, x_{2} x_{4}^{2}-x_{3}^{3}\right), \\
& \mathfrak{p}_{Y}=\left(x_{1}, x_{4}\right), \mathfrak{p}_{C}=\left(x_{1}, x_{2}, x_{3}, x_{4}\right) .
\end{aligned}
$$

Van der Waerden proves by a difficult method that $i(X, Y ; C)=4$. Taking the length of $A /\left(\mathfrak{p}_{X}+\mathfrak{p}_{Y}\right) A$ we get 5 . Using the Theorem we obtain

$$
\begin{aligned}
i(X, Y ; C) & =e_{0}\left(\left(x_{1}, x_{4}\right), A / \mathfrak{p}_{X}\right) \\
& =l\left(K\left[x_{0}, \ldots, x_{4}\right]_{\left(x_{1}, \ldots, x_{4}\right)} /\left(x_{1}, x_{4}, x_{2}^{2}, x_{2} x_{3}, x_{3}^{3}\right)\right)=4
\end{aligned}
$$


since, as is not hard to show, $U\left(\mathfrak{p}_{X}+\left(x_{1}\right)\right)=\left(x_{1}, x_{2}^{2}, x_{2} x_{3}, x_{2} x_{4}^{2}-x_{3}^{3}\right)$.

(3) The statement of the Lemma is true for $n=1$ if $A$ is a Buchsbaum ring (see e.g. [13], [14], [15]). This follows from Lemma 11 and Corollary 13 of [13].

(4) The statements of the Theorem and Proposition 1 are not true in general if we replace the definition of $U(\mathfrak{a})$ by $U i(\mathfrak{a})=\cap q$ where $q$ runs through the minimal primary ideals belonging to a. To see this take $A=$ $K[[x, y, z]] /(x y, x z)$ over any field $K$ and $q=(y, x+z) A$.

(5) We relate our observations to some global questions by considering the (affine) cones over projective varieties. We will give a numerical criterion for determining whether a variety is arithmetically Cohen-Macaulay (see also [8]).

We consider the polynomial ring $R=K\left[x_{0}, \ldots, x_{n}\right]$ in $n+1$ indeterminates over an arbitrary field $K$. Let a be a homogeneous ideal in $R$ of $h$-dimension $d \geqslant 0$; that is, (Krull) dimension of a in $R$ minus 1 . The degree of the ideal $\mathfrak{a}$ is denoted $h_{0}(\mathfrak{a})$. The extended ideal of $\mathfrak{a}$ in $R\left[x_{n+1}\right]$ is denoted $\mathrm{a}^{*}$. If $\mathrm{q} \subset R$ is a primary ideal belonging to $\left(x_{0}, \ldots, x_{n}\right)$ then $\mathrm{q}^{*}$ has $h$-dimension zero and $h_{0}\left(\mathrm{q}^{*}\right)>0$ in $R\left[x_{n+1}\right]$. We note that $h_{0}(\mathrm{a})=h_{0}\left(\mathrm{a}^{*}\right)$ if a has $h$-dimension $d \geqslant 0$ (see e.g. [4, 143.12]). Now we end with our application.

Proposition 3. The following statements are equivalent:

(a) a is perfect.

(b) There are forms $F_{1}, \ldots, F_{d+1}$ in $R$ such that $a+\left(F_{1}, \ldots, F_{d+1}\right)$ is a primary ideal belonging to $\left(x_{0}, \ldots, x_{n}\right)$ and

$$
h_{0}\left(\left(\mathfrak{a}+\left(F_{1}, \ldots, F_{d+1}\right)\right)^{*}\right)=h_{0}\left(a_{d+1}^{*}\right) .
$$

Proof. If $a$ is perfect then the assertion (b) is trivial. Suppose now that (b) is true. The first claim in our proof of Bezout's Theorem provides

$$
h_{0}\left(\left(\mathfrak{a}+\left(F_{1}, \ldots, F_{d+1}\right)\right)^{*}\right)=h_{0}(\mathfrak{a}) \cdot h_{0}\left(\left(F_{1}, \ldots, F_{d+1}\right)\right) .
$$

The Theorem of [8] shows that this equality is equivalent with (a). Q.E.D.

\section{REFERENCES}

1. M. Auslander and D. A. Buchsbaum, Codimension and multiplicity, Ann. of Math. (2) 68 (1958), 625-657.

2. E. Boda, Zur Berechnung von Schnittmultiplizitäten durch Längen, Mathematica Slovaca 28 (1978), 173-179.

3. Nguyen Tu Cuong, Peter Schenzel und Ngo Viet Trung, Verallgemeinerte CohenMacaulay-Moduln, Math. Nachr. (to appear).

4. W. Gröbner, Moderne algebraische Geometrie, Springer-Verlag, Wien und Innsbruck, 1949.

5. F. S. Macaulay, The algebraic theory of modular systems, Cambridge Univ. Press, London, 1916.

6. D. Mumford, Algebraic geometry. I, Springer-Verlag, Berlin and New York, 1976.

7. D. G. Northcott, Lessons on rings, modules and multiplicities, Cambridge Univ. Press, Cambridge, 1968.

8. B. Renschuch und W. Vogel, Zum Nachweis arithmetischer Cohen-Macaulay-Varietäten, Monatsh. Math. 85 (1978), 201-210.

9. M. Reufel, Beiträge zur Multiplizitäten und Spezialisierungstheorie. I, Gesellsch. Math. Datenverarbeitung Bonn, Ber. No. 57, Bonn, 1972, pp. 177-201.

10. P. Samuel, Méthodes d'algèbre abstraite en géométrie algébrique, Ergbnisse der Math., H.4, Springer-Verlag, Berlin and New York, 1955. 
11. J.-P. Serre, Algèbre locale. Multiplicités, Lecture Notes in Math., vol. 11, Springer-Verlag, Berlin and New York, 1965.

12. J. Stückrad und W. Vogel, Ein Korrekturglied in der Multiplizitätstheorie von D. G. Northcott und Anwendungen, Monatsh. Math. 76 (1972), 264-271.

13. __ Eine Verallgemeinerung der Cohen-Macaulay Ringe und Anwendungen auf ein Problem der Multiplizitätstheorie, J. Math. Kyoto Univ. 13 (1973), 513-528.

14. __ Über das Amsterdamer Programm von W. Gröbner und Buchsbaum Varietäten, Monatsh. Math. 78 (1974), 433-445.

15. __, Toward a theory of Buchsbaum singularities, Amer. J. Math. 100 (1978), 727-746.

16. B. L. van der Waerden, Eine Verallgemeinerung des Bezoutschen Theorems, Math. Ann. 99 (1928), 497-541.

17. A. Weil, Foundation of algebraic geometry, 2nd ed., Amer. Math. Soc. Colloq. Publ. no. 29, Amer. Math. Soc., Providence, R. I., 1962.

18. D. J. Wright, A characterisation of multiplicity, Monatsh. Math. 79 (1975), 165-167.

19. O. Zariski and P. Samuel, Commutative algebra. Vol. II, Princeton Univ. Press, Princeton, N. J., 1962.

FAculty of Sciences, Department of Geometry, University of Komenský, 81631 Bratislava, Czechoslovakia

Department of Mathematics, Martin-Luther-University, 401 Halle, German DeMOCRATIC REPUBLIC 